\title{
Pathology of astrovirus associated diarrhoea in a paediatric bone marrow transplant recipient
}

\author{
N J Sebire, M Malone, N Shah, G Anderson, H B Gaspar, W D Cubitt
}

J Clin Pathol 2004;57:1001-1003. doi: 10.1136/icp.2004.017178

\begin{abstract}
Human astrovirus infection often causes outbreaks of self limiting diarrhoea, but may also infect patients who are immunodeficient or immunocompromised. Although there are previous publications relating to various aspects of astroviruses, there is a minimal amount of literature on the histopathological features of gastrointestinal astrovirus infection in humans. We report the histopathological findings, including immunohistochemical and electron microscopic features, of astrovirus infection in a bone marrow transplant recipient aged 4 years with diarrhoea. The appearance of a small intestinal biopsy did not suggest graft versus host disease, but demonstrated villous blunting, irregularity of surface epithelial cells, and an increase in lamina propria inflammatory cell density. Immunohistochemical staining with a murine astrovirus group specific monoclonal antibody demonstrated progressively more extensive staining in the duodenal and jejunal biopsies, predominantly restricted to the luminal surface and cytoplasm of surface epithelial cells, most marked at the villus tips. Electron microscopic examination demonstrated viral particles within the cytoplasm of enterocytes, focally forming paracrystalline arrays.
\end{abstract}

A stroviruses-single stranded RNA viruses-were first detected by Appleton and Higgins in 1975 as the causative agent of an outbreak of diarrhoea and vomiting in a maternity unit in Brighton, UK. ${ }^{1}$ Subsequently, astroviruses were found in the faeces of lambs, ${ }^{2}$ calves, ${ }^{3}$ and piglets. ${ }^{4}$ In recent years, the application of reverse transcription polymerase chain reaction and commercial enzyme immunoassays ${ }^{56}$ for the examination of faecal extracts has established that human astroviruses are an important cause of diarrhoea and vomiting in infants and the elderly. Astrovirus often infects patients with human immunodeficiency virus ${ }^{7}$ and patients who are immunodeficient or immunocompromised. ${ }^{8}{ }^{9}$

Although there are several publications relating to various aspects of astroviruses, there is only one brief previous report of the histopathological features of astrovirus infection in humans, which showed the presence of particles in a duodenal biopsy. ${ }^{10}$ Studies in experimentally infected lambs ${ }^{11}$ showed that astroviruses infected only mature enterocytes and subepithelial macrophages in the small intestine, resulting in villus atrophy. Enterocytes containing intracytoplasmic vacuoles and inclusions and degenerate nuclei were seen. Electron microscopic examination revealed the presence of virus particles, sometimes in paracrystalline arrays, along the microvilli and in lysosomes and autophagic vacuoles; virus particles were also detected in lysosomes in macrophages in the lamina propria. In contrast, the bovine virus was found preferentially to infect the epithelium covering the dome villi of the jejunal and ileal Peyers patches. ${ }^{12}$ Immunofluorescence and electron microscopic examination revealed infection in $\mathrm{M}$ cells and in absorptive enterocytes of the dome villi; exudates of sloughed epithelial cells were also seen. A recent study of astrovirus infection in young turkeys ${ }^{13}$ has shown that the virus may induce diarrhoea in the apparent absence of histological inflammation and cell death. The authors postulated that there may be a viraemic stage during infection because virus was recovered from multiple tissues, and suggested that the lack of an inflammatory response may be the result of increased activation of the cytokine transforming growth factor $\beta$ during viral infection.

\section{"Astrovirus often infects patients with human immunode- ficiency virus and patients who are immunodeficient or immunocompromised"}

In our study, we report for the first time the histopathological features, including immunohistochemical and electron microscopic findings, of gastrointestinal astrovirus infection in a bone marrow transplant recipient aged 4 years with diarrhoea.

\section{CASE REPORT}

The patient was a 4 year old boy who initially presented at the age of 1 month with a history of mouth ulcers and failure to thrive. Following colonoscopy and colectomy, he was diagnosed with intractable ulcerating enterocolitis of infancy, which is characterised by onset in the lst week of life, a Crohn's disease-like enterocolitis with marked oro-anal involvement, and unresponsiveness to immunosuppressive treatment. ${ }^{14}$ There is a high incidence of the development of Epstein Barr virus driven lymphoma, and bone marrow transplant was shown to have a beneficial effect on the patient's older brother, also affected by the same condition. He was maintained on immunosuppressive and immunoglobulin replacement treatment, but at the age of 4 years he also received a bone marrow transplant from a matched unrelated donor following fludarabine/melphalan conditioning. Routine weekly screening of stools (ileostomy fluid) by electron microscopy after the transplant first demonstrated gastrointestinal excretion of astrovirus on day 30, following which he continued to excrete virus for 62 days. Administration of oral immunoglobulin had no apparent effect on the viral load. The identification of astrovirus in all the electron microscopy positive samples was confirmed by enzyme immunoassay (Astro IDEIA; Dako, Ely, Cambridgeshire, UK). An endoscopic gastrointestinal biopsy series was carried out 10 weeks post-transplant to determine whether the cause of the diarrhoea was graft versus host disease (GVHD) or intestinal viral infection. The gastric biopsy was unremarkable. The duodenal and jejunal biopsies did not show features suggestive of GVHD, but demonstrated mild to moderate architectural alteration with villus blunting

Abbreviations: GVHD, graft versus host disease 
and irregularity of surface epithelial cells. There was no histologically active inflammation and no viral inclusion bodies were seen, but there was an increase in lamina propria inflammatory cell density, predominantly composed of eosinophils with scattered polymorphs, most pronounced in the duodenal biopsy (fig lA). Immunohistochemical staining for adenovirus was negative in all biopsies, although the patient was shown by polymerase chain reaction to have a concurrent adenovirus viraemia. However, immunohistochemical staining with a murine astrovirus group specific monoclonal antibody (8E7), ${ }^{15}$ using a standard protocol ${ }^{16}$ at a 1/100 dilution with an avidin-biotin based detection system following protease antigen retrieval, demonstrated negative staining in the gastric biopsy, but progressively more extensive staining in the duodenal and jejunal biopsies (fig $1 \mathrm{~B}, \mathrm{C}$ ) Immunostaining was predominantly restricted to the lumenal surface and the cytoplasm of the surface epithelial cells, and was most pronounced at the tips of the villi (staining of a normal control small intestinal biopsy was entirely negative, as was retrospective immunostaining of pretransplant biopsies from the index patient). Electron microscopic examination of a paraffin wax embedded small bowel biopsy, reprocessed for ultrastructural studies, demonstrated numerous viral particles within the cytoplasm of enterocytes, focally forming paracrystalline arrays (fig 1D).

\section{DISCUSSION}

Our study demonstrates an association between astrovirus infection of the proximal small intestine and the appearance of pronounced diarrhoea in an immunocompromised patient and, furthermore, reports the histopathological features of gastrointestinal astrovirus infection in humans in this setting, including immunohistochemical and ultrastructural findings.

Despite severe diarrhoea, the morphological abnormalities present were relatively minor and non-specific; in particular, the inflammatory response was only mild. It could be argued that because the patient was immunocompromised, the tissue reaction may be modified. This of course is true, but in this case there was good evidence of engraftment and other acute and chronic inflammatory reactions are well recognised in many other complications occurring in transplant recipients. It is most likely that the pathogenesis of astrovirus associated diarrhoea is not primarily inflammatory in nature, a finding in accordance with a recent study examining turkeys infected with astrovirus. ${ }^{13}$ An additional study, also examining turkeys, showed only slight evidence of enteric damage with astrovirus infection, which was characterised by mild epithelial necrosis, a lamina propria inflammatory infiltrate, minimal villus atrophy, and mild crypt hyperplasia, morphological findings very similar to those in our present case. ${ }^{17}$ In addition, a study of poults inoculated with astrovirus reported that they developed diarrhoea, which was associated with only minor histological changes throughout the small intestine, but with a significant reduction in intestinal specific maltase activity, which may have resulted in disaccharide maldigestion, malabsorption, and osmotic diarrhoea. As astrovirus was cleared from the intestinal tract, maltase activity was restored and the diarrhoea resolved..$^{18}{ }^{19}$ The distribution of infected cells in our case was also similar to that previously described in lambs, in which infection involved mature columnar epithelial cells covering the apical two thirds of the small intestinal villi, with virus particles released by desquamated cells into the gut lumen. ${ }^{3}$

In our present case, there was evidence of small intestinal, but not gastric, involvement by astrovirus, with more extensive infection in the jejunal compared with the duodenal biopsies. At all sites in which immunohistochemical staining was positive, the only significantly infected cells appeared to be surface enterocytes, particularly those near the villus tips. This apparent pronounced cellular tropism is consistent with previous reports of astrovirus infection in animals, which also suggested preferential involvement of small intestinal enterocytes. It is likely that astrovirus undergoes adsorbtive endocytosis and internalisation at the site of coated pits, but the exact mechanism of cellular entry remains unclear.
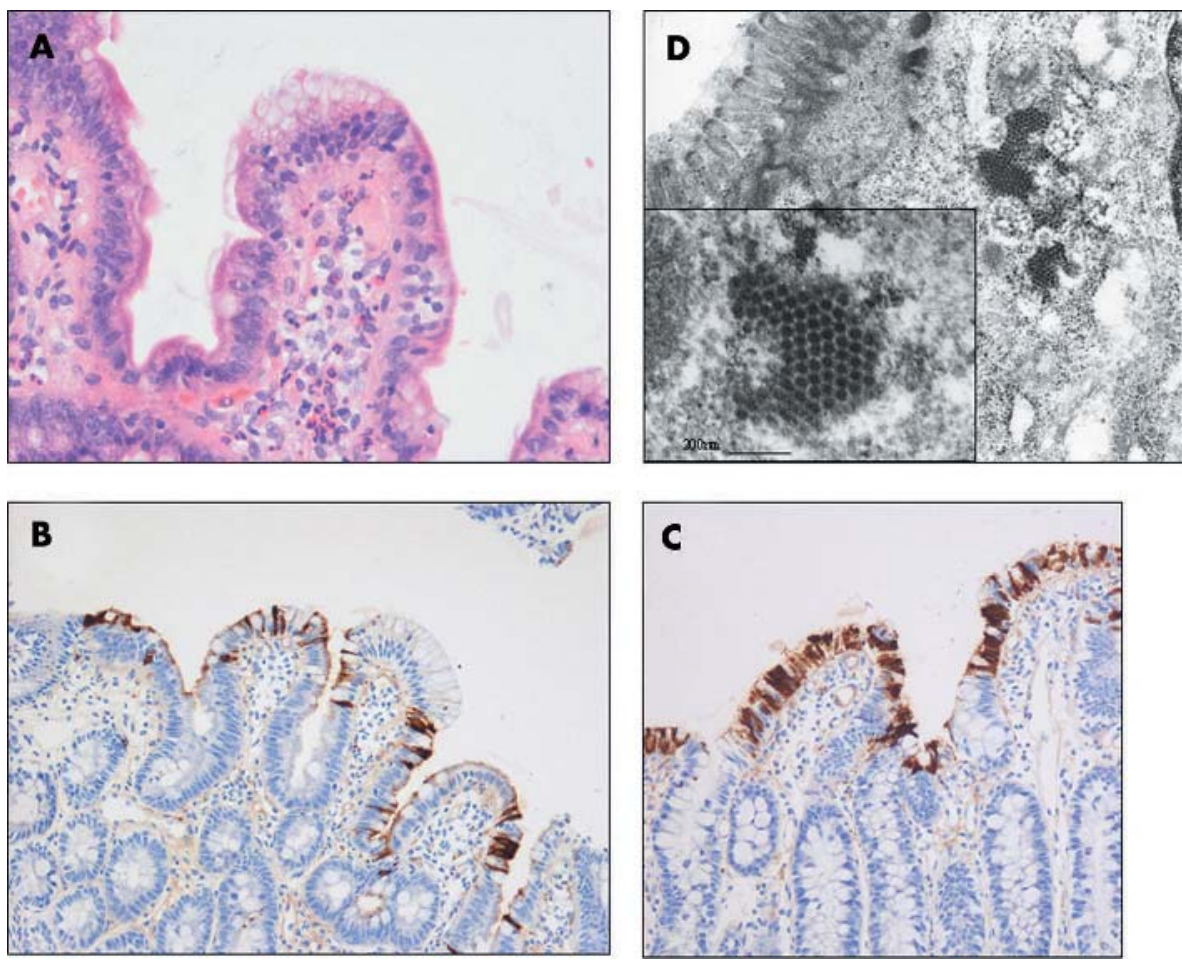

Figure 1 (A) Photomicrograph of a jejunal biopsy specimen from a bone marrow transplant recipient with astrovirus infection demonstrating villus blunting, non-specific alterations in surface epithelial cells, and a mixed lamina propria inflammatory infiltrate, but without the presence of viral inclusion bodies (original magnification, $\times 100$ ).

Photomicrographs of (B) duodenal and (C) jejunal biopsies from a bone marrow transplant recipient with astrovirus infection immunostained with anti-astrovirus antibody and demonstrating progressively more extensive staining of surface epithelial cells, most commonly near the villus tips (original magnifications, $\times \mathbf{4 0}$ and $\times 100$, respectively). (D) Electron micrographs of a jejunal enterocyte demonstrating cytoplasmic paracrystalline viral arrays of astrovirus (original magnifications, $\times 32000$ and $\times 100000$ (inset)). 


\section{Take home messages}

- We describe the histopathological features of human gastrointestinal astrovirus infection, including immunohistochemical and electron microscopic findings, in an immunocompromised child

- Human astrovirus infection in the gastrointestinal tract may demonstrate a non-specific mild enteropathy and requires additional ancillary techniques to provide a definite diagnosis

- Astrovirus infection may be of clinical relevance in immunosuppressed patients and should be considered in the differential diagnosis of diarrhoea in such cases

Because immunohistochemical staining on paraffin wax embedded tissues for astrovirus has not been reported before, it was important to confirm that such staining represented true cellular infection, rather than either non-specific staining, cross reactivity, or the detection of bare viral protein. Therefore, electron microscopy was used as a confirmatory technique and revealed large numbers of intracytoplasmic particles, focally forming paracrystalline arrays, as previously described in animal inoculum studies.

"The distinction between graft versus host disease and infection is a relatively common, but difficult, clinical problem in bone marrow transplant and small bowel transplant recipients"

The histopathological findings of GVHD in intestinal biopsies may be subtle, with focal basal epithelial vacuolisation or apoptosis and an increase in intraepithelial lymphocytes. ${ }^{20}$ The distinction between GVHD and infection is a relatively common, but difficult, clinical problem in bone marrow transplant and small bowel transplant recipients. The most common viral infection in this setting is adenovirus infection. In the absence of known astrovirus infection on the basis of stool virology, in our case the histopathological findings were subtle and apparently non-specific, and the diagnosis of astrovirus infection would have been impossible on histological examination alone. Immunohistochemical staining, even in paraffin wax embedded sections, appears to be a useful technique in such cases, but prospective studies of its more widespread use in immunocompromised patients are required before sensitivities for detection of infection can be determined.

Astrovirus infection may be a cause of diarrhoea in immunocompromised children and can be diagnosed on intestinal biopsies. The histopathological features are nonspecific, but in conjunction with immunohistochemistry and/ or electron microscopy, a definitive diagnosis may be made.

\section{Authors' affiliations}

N J Sebire, M Malone, G Anderson, Department of Histopathology, Great Ormond Street Hospital, Great Ormond Street, London WCIN 3JH, UK

N Shah, Department of Gastroenterology, Great Ormond Street Hospital, Great Ormond Street, London

H B Gaspar, Department of Immunology, Great Ormond Street Hospital W D Cubitt, Department of Virology, Great Ormond Street Hospital, Great Ormond Street

Correspondence to: Dr N J Sebire, Department of Histopathology, Great Ormond Street Hospital, Great Ormond Street, London WCIN 3JH, UK; SebirN@gosh.nhs.uk

Accepted for publication 15 March 2004

\section{REFERENCES}

1 Appleton H, Higgins PG. Viruses and gastroenteritis in infants. Lancet 1975;1:1297.

2 Snodgrass DR, Gray EW. Detection and transmission of $30 \mathrm{~nm}$ virus particles (astroviruses) in faeces of lambs with diarrhoea. Arch Virol 1977;55:287-91.

3 Gray EW, Angus KW, Snodgrass DR. Ultrastructure of the small intestine in astrovirus-infected lambs. J Gen Virol 1980;49:71-82.

4 Bridger JC. Detection by electron microscope of caliciviruses, astroviruses and rotavirus-like particles in the faeces of piglets with diarrhoea. Vet Res 1980; 107:532-3.

5 Cubitt WD. Historical background and classification of caliciviruses and astroviruses. Arch Virol 1996;12:225-5.

6 Mitchell DK. Astrovirus gastroenteritis. Pediatr Infect Dis J 2002;21:1067-9.

7 Grohmann GS, Glass RI, Pereira HG, et al. Enteric viruses and diarrhoea in HIV-infected patients. N Engl Med J 1993;329:14-20.

8 Cubitt WD, Mitchell DK, Carter MJ, et al. Application of electron microscopy, enzyme immunoassay, and RT-PCR to monitor an outbreak of astrovirus type 1 in a paediatric bone marrow transplant unit. J Med Virol 1999;57:313-21.

9 Coppo P, Scieux C, Ferchal F, et al. Astrovirus enteritis in a chronic lymphocytic leukemia patient treated with fludarabine monophosphate. Ann Hematol 2000;79:43-5.

10 Phillips AD, Rice SJ, Walker-Smith JA. Astrovirus within small intestinal mucosa. Gut 1982;23:A923-4.

11 Hall GA. Comparative pathology of infection by novel diarrhoea viruses. Ciba Found Symp 1987; 128:192-217

12 Woode GN, Pohlenz JF, Kelso-Gourley NE, et al. Astrovirus and Breda virus infections of dome cell epithelium of bovine ileum. J Clin Microbiol 1984; 11:441-52.

13 Koci MD, Moser LA, Kelley LA, et al. Astrovirus induces diarrhea in the absence of inflammation and cell death. J Virol 2003;77:1 1798-808.

14 Sanderson IR, Risdon RA, Walker-Smith JA. Intractable ulcerating enterocolitis of infancy. Arch Dis Child 1991;66:295-9.

15 Herrmann JE, Nowak NA, Perron-Henry DM, et al. Diagnosis of astrovirus gastroenteritis by antigen detection with monoclonal antibodies. J Infect Dis 1990;161:226-9.

16 Miller K. Immunocytochemical techniques. In: Bancroft JD, Gamble M, eds. Theory and practice of histological techniques. London: Churchill Livingstone, 2002:421-64.

17 Behling-Kelly E, Schultz-Cherry S, Koci M, et al. Localization of astrovirus in experimentally infected turkeys as determined by in-situ hybridization. Vet Pathol 2002;39:595-8.

18 Thouvenelle ML, Haynes JS, Reynolds DL. Astrovirus infection in hatchling turkeys: histologic, morphometric, and ultrastructural findings. Avian Dis 1995;39:328-36.

19 Thouvenelle ML, Haynes JS, Sell JL, et al. Astrovirus infection in hatchling turkeys: alterations in intestinal maltase activity. Avian Dis 1995;39:343-8.

20 Snover DC, Weisdorf SA, Vercellotti GM, et al. A histopathologic study of gastric and small intestinal graft-versus-host disease following allogeneic bone marrow transplantation. Hum Pathol 1985;16:387-92. 